\title{
Cognition, daily living, and health-related quality of life in 85-year-olds in Sweden
}

\author{
Maria M. Johansson, Ewa Wressle and Jan Marcusson
}

\section{Linköping University Post Print}

N.B.: When citing this work, cite the original article.

This is an electronic version of an article published in:

Maria M. Johansson, Ewa Wressle and Jan Marcusson, Cognition, daily living, and healthrelated quality of life in 85-year-olds in Sweden, 2012, Aging, Neuropsychology and Cognition, (19), 3, 421-432.

Aging, Neuropsychology and Cognition is available online at informaworld ${ }^{\mathrm{TM}}$ :

http://dx.doi.org/10.1080/13825585.2011.629290

Copyright: Taylor \& Francis (Psychology Press): STM, Behavioural Science and Public Health Titles / Taylor \& Francis (Psychology Press)

http://www.psypress.com/

Postprint available at: Linköping University Electronic Press

http://urn.kb.se/resolve?urn=urn:nbn:se:liu:diva-77575 


\section{Cognition, daily living, and health-related quality of life in 85-year-olds in Sweden}

Authors: Maria Johansson, BScOT, Division of Geriatrics, Dept. of Clinical and Experimental Medicine, Faculty of Health Sciences, Linköping University, Dept. of Geriatric Medicine, County Council of Östergötland, Linköping, Sweden

Jan Marcusson, Professor, Division of Geriatrics, Dept. of Clinical and Experimental Medicine, Faculty of Health Sciences, Linköping University, Dept. of Geriatric Medicine, County Council of Östergötland, Linköping, Sweden

Ewa Wressle, Associate prof, Division of Geriatrics, Dept. of Clinical and Experimental Medicine, Faculty of Health Sciences, Linköping University, Dept. of Geriatric Medicine, County Council of Östergötland, Linköping, Sweden

Corresponding author: Ewa Wressle

Dept. of Geriatric Medicine

University Hospital

SE-581 85 Linköping, Sweden

Phone: +46101034110

Fax: +46101034141

E-mail: Ewa.Wressle@lio.se 


\begin{abstract}
This study investigates how cognition influences activities of daily living and healthrelated quality of life in 85-year-olds in Sweden $(n=373)$. Data collection included a postal questionnaire comprising demographics and health-related quality of life measured by the EQ-5D. The ability to perform personal activities of daily living was assessed during a home visit that included administering the Mini Mental State Examination (MMSE). Cognitive impairment was shown in 108 individuals (29\%). The majority were independent with respect to personal activities of daily living (PADL). A larger number of participants with cognitive impairment reported that they needed assistance in instrumental activities of daily living (IADL) compared to the group without cognitive impairment. Impaired cognition was significantly related to problems with IADL. Significant but low correlations were found between cognition and health-related quality of life - higher ratings on perceived quality of life correlated with higher results on the MMSE.
\end{abstract}




\section{INTRODUCTION}

Sweden has the highest percentage of 80 year olds or older in the world (Larsson \& Thorslund, 2006). Since this age group is continuously increasing, attention should be drawn to the consequences of this fact. Impairment of health status increases with age and more than two-thirds of people aged 85+ has impaired health status (König, Heider, Lehnert, RiedelHeller, Angermeyer, Matschinger et al., 2010). For example, in an elderly population in Brazil, cognitive performance has been used as a predictor of functional incapacity for activities of daily living (Cruz Moreira de Castro \& Oliveira Guerra, 2008). Elderly individuals' ability to manage activities of daily living (ADL) decreases with age, but individual differences exist (Demura, Sato, Minami, \& Kasuga, 2003). Minimal cognitive impairment increases the risk of future dysfunction in ADL and in instrumental activities of daily living (IADL). This increase in risk has implications for health care such as need for regular assessment of physical conditions and reduction of hypertension to reduce stroke incidence (Dodge, Kadowaki, Hayakawa, Yamakawa, Sekikawa, \& Ueshima, 2005). Early detection of cognitive decline could lead to secondary prevention as this information could be used to develop strategies that control risk factors; therefore, early detection of cognitive decline should be systematically assessed by clinicians (Gauthier, Reisberg, Zaudig, Petersen, Ritchie, Broich et al., 2006).

Basic ADL remain preserved when the first symptoms of cognitive deterioration occurs whereas the ability to perform complex ADL are more likely to decrease when memory, attention, and executive functions deteriorate (Perneczky et al., 2006b). It is important to assess both cognitive function and the ability to perform complex ADL in dementia investigations (Perneczky et al., 2006b; Nygård, Amberla, Bernspång, Almkvist, \& Winblad, 1998). Mild Cognitive Impairment (MCI) negatively influences an individual's ability to perform complex ADL (Aretouli \& Brandt, 2010; Burton, Strauss, Hunter, \& Hultsch, 2009; Allaire, Gamaldo, Ayotte, Sims, \& Whitfield, 2009; Perneczky et al., 2006a) and increases the risk for dementia (Palmer, Fratiglioni, \& Winblad, 2003). Performing tasks at slower speeds might be an early indicator of functional change in MCI (Wadley, Okonkwo, Crowe, \& RossMeadows, 2008). Gender differences have been shown: men with memory problems and women with poor cognitive performance reported more problems in IADL compared to those without memory problems or poor cognitive performance, respectively (Avlund \& Fromholt, 1998). Compared to men, more women had disability in ADL and IADL and lower cognitive function (Polidoro, Dornbusch, Vestri, Di Bona, \& Alessandri, 2011). 
The Swedish Umeå 85+ study showed that depression is associated with dependency in personal ADL and a lower score on the MMSE (Bergdahl, Gustavsson, Kallin, von Heideken Wågert, Lundman, Bucht et al., 2005). The Kungsholmen Project in Stockholm, Sweden, provided evidence that impaired cognition affects performance in IADL whereas ADL is affected when a certain degree of cognitive dysfunction is reached (Agüero-Torres, Thomas, Winblad, \& Fratiglioni, 2002). A substantial acceleration of cognitive decline appears several years before diagnosis of dementia; results found in general Swedish populations in Kungsholmen and in Gothenburg (Thorvaldsson, MacDonald, Fratiglioni, Winblad, Kivipelto, Jonsson Laukka, \& et al., 2011). Thus, early detection of cognitive dysfunction is of outmost importance in primary care. In addition, measurements of ADL and IADL should be performed regularly in the elderly population in order to know when and how to intervene.

Most published studies regarding cognitive function, or rather dysfunction, focus on individuals with a diagnosis of dementia or MCI. Moreover, these studies do not focus on a population of elderly people and do not consider the effects on health-related quality of life. To fill this knowledge gap, we investigated the relationships between cognitive function, ability to perform activities of daily living and perceived health-related quality of life in 85year-old individuals in Sweden.

\section{METHODS}

This study is part of the Elderly in Linköping Screening Assessment (ELSA 85), a population study of 85-year-olds in the southern Sweden (Nägga et al., 2011). ELSA 85 characterized and defined evidence-based knowledge on how best to plan, design, and provide health care for the oldest-old. The study focused on physical, cognitive, and environmental factors in 85-year-old individuals and their ability to perform meaningful activities. The present study was approved by the Research Ethics Committee of Linköping University, Sweden (141-06). Written informed consent was obtained from all participants and they were told that participation was voluntary and confidential.

\section{Sample}

All persons born in 1922 and living in Linköping municipality in Sweden $(n=650)$ received a postal questionnaire that included information about the study and options for participation in each phase of the study or the choice not to participate at all. Of these 650 
individuals, 586 (90\%) replied and 496 (76\%) provided written informed consent and answered the postal questionnaire. Drop-outs $(n=52)$ were individuals that we could not contact by post or telephone. Twelve individuals had died. All individuals who answered the questionnaire and expressed an interest in participating in the study were included in the next phase, a home visit by an occupational therapist. The occupational therapist interviewed the participants to gather information about their cognitive abilities and their activities of daily living. In total, 380 individuals $(76 \%$ of the individuals who answered the postal questionnaire) agreed to a home visit. Cognitive assessment was performed in 373 cases with an internal drop-out of seven cases. Thus, all results are calculated on 373 individuals.

Comparisons between non-participants $(n=277)$ and participants $(n=373)$ revealed a statistically significant difference in type of accommodation $(\mathrm{p}<.001)$ and in gender $(\mathrm{p}=$ .001). In the participant group, a significantly larger number of individuals lived in ordinary housing and a smaller number in nursing homes. More men than women agreed to participate.

\section{Postal questionnaire}

The postal questionnaire included questions about demographics, education, socioeconomic status, occupation, social network, and use of assistive technology. Socioeconomic status (SES), referring to the elderly's previous occupation, was classified into the following categories: low (blue collar); intermediate (white collar); and high (self-employed or academic profession) (Dutton \& Levine, 1989). In addition, the EQ-5D (EuroQol Group, 1990; Brooks, 1996; Rabin \& de Charro, 2001) - a generic instrument that assesses healthrelated quality of life in terms of mobility, self-care, usual activities, pain/discomfort, and anxiety/depression - was used. The EQ-5D response alternatives are no problems, moderate problems, or extreme problems. The scores on the five EQ-5D items were converted into a single summary index value generated by means of the Time-Trade-Off method (TTO) (Rabin \& de Charro, 2001; Dolan, 1997). The EQ-5D index value ranges from +1 to -1 , where +1 represents perfect health, 0 a state equivalent to death, and -1 worse than death (Dolan, 1997). A visual analogue scale (VAS) recording the individual's self-rated valuation of health is included, ranging from 0 (worst imaginable health state) to 100 (best imaginable health state). The EQ-5D is considered to be a practical and easy to administer tool for assessing the elderly population (Holland, Smith, Harvey, Swift, \& Lenaghan, 2004), valid in a general population (Johnson \& Pickard, 2000), and effective in persons with cognitive impairments (Wolfs, Dirksen, Kessels, Willems, Verhey, \& Severens, 2007). 


\section{Assessments performed at the home-visit}

Incomplete answered postal questionnaires were run through with the participants at the home-visit. Ability to perform personal activities of daily living (PADL) was assessed using four questions that addressed the participant's ability to perform the following activities: dressing and undressing; bathing; toileting; and eating. Answer alternatives were independent, need of some help, or need of much help. The Instrumental Activity Measure (IAM) (Grimby G et al., 1996; Andrén et al., 1997; Andrén \& Grimby, 2000; Daving et al., 2008) was used to assess dependence and perceived difficulty in instrumental activities of daily living (IADL) in eight items (locomotion outdoors, simple meal, cooking, public transportation, small-scale shopping, large-scale shopping, cleaning, and washing) with the following scoring alternatives: $4=$ no problems; $3=$ some problems; 2 = great problems; and $1=$ impossible.

Cognitive function was assessed using the Mini Mental State Examination (MMSE) (Folstein, Folstein, \& McHugh, 1975). The MMSE assesses orientation to time and place, attention, memory, and language and visual construction. The MMSE has a maximum of 30 points where higher scores indicate better cognition. Recent recommended cut-off levels were used: $\geq 27=$ no impairment; $21-26=$ mild; $11-20=$ moderate; and $\leq 10=$ severe impairment (Folstein, Folstein, McHugh, \& Fanjiang, 2001).

\section{Statistical analyses}

Statistical non-parametric analyses were performed using the PASW 18.0 Statistical package. The results from the EQ-5D items were dichotomized into two categories: being independent/having no problems, no pain/discomfort, no anxiety/depression or being in need of help/having problems, pain, worries. The P-ADL items were dichotomized into two categories: being independent or being in need of help. The IAM items were dichotomized into two categories: having problems or having no problems. For comparative analyses, participants were divided into three groups regarding MMSE scores: moderate/severe impairment (0-20); mild impairment (21-26); and no impairment (27-30). Comparisons were also performed between participants with cognitive impairment (MMSE 0-26) and participants without cognitive impairment (MMSE 27-30). Regarding differences between the groups based on MMSE scores, the Chi-squared test was used for categorical data. The MannWhitney U-test was used for ordinal scales (MMSE and EQ-5D items) as well as for the EQ5D index value and VAS as data was not normally distributed. A p-value $<0.05$ was 
considered statistically significant.) Spearman's rank-order correlation was employed for analyses of associations between cognition revealed by the MMSE, years of education, and health-related quality of life as found in the EQ-5D VAS and EQ-5D index value.

\section{RESULTS}

\section{Cognition and health-related quality of life}

The mean value for MMSE was 27 ( $\mathrm{SD}=3.385$ ) for all participants, ranging from six to 30, and no difference was found with respect to gender. There were cognitive impairments for $43(12 \%)$ individuals when using the traditional cut off for cognitive impairment $(<24)$ on the MMSE. When using the cut off of <27 points, 108 (29\%) individuals had cognitive impairment. In 14 cases (4\%), a dementia diagnosis existed.

Perceived health assessed by EQ-5D showed that $50 \%$ of all participants had no mobility problems, $85 \%$ were independent in self-care, $74 \%$ managed their usual activities, $67 \%$ perceived pain/discomfort, and 35\% had anxiety/depression. The scoring on the EQ-5D visual analogue scale for perceived health revealed a mean value of $67(\mathrm{SD}=19.8)$ and ranged from 0 to 100 . The mean score of the EQ-5D index value was 0.72 (SD 0.24) and ranged from -0.181 to 1.00 .

Table 1 presents the characteristics of participants in relation to MMSE scores: moderate/severe impairment (0-20); mild impairment (21-26); and no impairment (27-30). Lower socioeconomic status was more common in participants experiencing impaired cognition, living in community housing, requiring daily community assistance, requiring transportation service, and requiring a personal alarm. Mobility problems, need for help, presence of anxiety/depression, and lower health-related quality of life were reported significantly more by participants with impaired cognition. Statistical significant differences between the three groups were found regarding the MMSE areas: for language, $p=0.002$ and for all other MMSE areas, $\mathrm{p}<.001$.

\section{PADL and IADL}

The majority of all participants were independent in the four PADL items (84-99\%); only a few people needed help with bathing, dressing, toileting, or eating. Participants with impaired cognition (MMSE score 6-26) reported more problems with bathing (29\% vs. 9\%, p 
$<.001)$ and dressing (9\% vs. 5\%, p < .001) compared to the group without cognitive impairment (MMSE 27-30). Seven participants (6\%) with cognitive impairment had problems toileting compared to three participants without cognitive impairment (1\%); this was not statistically significant. Only two persons needed help eating, and both of these had cognitive impairments.

For all eight IADL items, a larger proportion of participants with cognitive impairment (MMSE 6-26) reported need of assistance compared to the group without cognitive impairment (MMSE 27-30): locomotion outdoors, $54 \%$ vs. $41 \%(\mathrm{p}=.027)$; simple meal, $34 \%$ vs. $9 \%$ (p <.001); cooking, $51 \%$ vs. $19 \%$ (p <.001); public transportation, $57 \%$ vs. $31 \%$ (p < .001 ); small-scale shopping, 55\% vs. $20 \%$ (p <.001); large-scale shopping, $65 \%$ vs. $33 \%$ (p < .001 ); cleaning, $71 \%$ vs. $48 \%$ (p < .001); and washing, $60 \%$ vs. 29 ( $p<.001$ ). Divided into three MMSE groups, all IADL items except locomotion outdoors showed significant differences between groups (Table 2).

\section{Correlation analyses}

There were significant but low correlation coefficients between EQ-5D index value and MMSE score $(r=0.145, \mathrm{p}<.001)$ and between EQ-5D VAS and MMSE score $(r=0.178, p$ .001). Higher ratings on perceived quality of life correlate with higher results on the MMSE. There was also a significant relationship between education years and MMSE score ( $\mathrm{r}=$ $0.192, \mathrm{p}<.001)$, indicating that more education correlates with better cognitive function. The correlation coefficient between socioeconomic status (SES) and MMSE was $r=0.259(\mathrm{p}<$ .001). Table 3 presents significant correlations between MMSE score and IADL items measured by the IAM - higher cognitive function associates to better ability to perform in all IADL items.

Correlation analyses between ADL items in the EQ-5D (mobility, self-care and usual activities) and PADL items showed significant relationships $(r=0.202-0.654, \mathrm{p}<.001)$ for all PADL items except eating $(\mathrm{r}=0.111-0.210, \mathrm{p}<.05)$. Correlations between the same three EQ-5D items and IAM items showed significant relationships with $r$ from -0.298 to $0.558(\mathrm{p}<.001)$. 


\section{DISCUSSION}

Our main finding in this general population is that IADL functions are negatively influenced in participants with cognitive impairment, even in mild impairment, while the capacity to perform PADL is less influenced. This is shown in earlier studies in MCI (Avlund \& Fromholt, 1998; Wadley et al., 2008; Allaire et al., 2009; Nygård et al., 1998; Nygård, 2003), in a cross-sectional study in Belgium (Kurz, Scuvee-Moreau, Vernooij-Dassen, Dresse, $\&$ the NADES Group, 2003), and in community-dwelling elders in Japan (Dodge et al., 2005). The results confirm that in addition to cognition, ADL (especially IADL) should be considered when assessing dementia. Structured assessments of both IADL and cognition could be performed as a screening in primary care to detect small cognitive deficits at an early stage and to initiate interventions aimed at improving and/or sustaining a person's activity level and thus quality of life.

Another important finding was that cognitive impairment, even mild impairment, was related to lower health-related quality of life. A Danish study in patients with dementia found that depending on others in ADL affected quality of life negatively (Andersen, WittrupJensen, Lolk, Andersen, \& Kragh-Sørensen, 2004). Since quality of life and ability to perform ADL are shown to be associated with Subjective Memory Complaints (SMC), this should be considered by health workers during daily consultations (Montejo, Montenegro, Fernández, \& Maestú, 2011). In a cross-sectional study, Waldorff and Rishøj (2008) confirmed the importance of asking patients about memory problems; they found that quality of life was the only predictor of self-reported memory problems.

The mean value for MMSE was rather high but participants' scores showed a wide range. One limitation of our study was the large proportion of non-participants living in sheltered housing compared to the participants. These non-participants probably had more significant cognitive impairments than the participants. We also had a gender difference; there were more men in the participant group than in the non-participant group. However, comparing the cognitive status between men and women did not show any gender difference, a finding that contradicts what Polidoro et al. found (2011).

The use of a postal questionnaire to this elderly population might raise questions. Ninety per cent of our population answered to the invitation letter, $76 \%$ participated in this phase. Due to practical implications we found a postal questionnaire suitable. A limitation might be that MMSE as a screening instrument is too insensitive. However, all MMSE areas differed between the three groups categorized according to MMSE scores, indicating that the 
instrument did detect cognitive impairments. The cut-off at $<27$ could also be questioned. We found significant differences between the groups concerning both IADL and health-related quality of life. The four open questions on PADL have not been tested regarding psychometric properties which is a limitation of this study. A more detailed and tested PADL instrument would have been helpful, yet this is not the primary area that reveals early problems with cognition. The significant correlations between ADL items in the EQ-5D and ADL items, and IADL items respectively, strengthen the validity of these data although the two different data collections methods (postal questionnaire and interview at home visit). Optimal data collection method should be observations but this is very time and resource consuming and probably would decrease the number of participants.

Future research should focus on development of a model for early detection of cognitive impairment including a vital area such as IADL. There are many instruments available and this large selection actually makes it problematic to compare individuals or groups of individuals and makes it difficult to determine what signs suggest cognitive impairments. A model that detects cognitive impairments must be easy to administer, not too time-consuming, rely on standardised instruments that are tested regarding psychometric properties, be helpful for further investigations, and guide the therapist to interventions aiming to prevent or improve cognitive functions.

\section{CONCLUSIONS}

Early detection of cognitive impairments is vital for establishing interventions aimed at reducing inactivity and improving or sustaining a person's activity level and thus quality of life. This is beneficial for the individual, society, and the health care system. The individual's capacity to perform IADL should be included in the assessment phase.

\section{ACKNOWLEDGMENTS}

This work was supported by grants from The Health Research Council of the SouthEast of Sweden (FORSS-8888, FORSS-11636, FORSS-31811), the County of Östergötland (LIO-11877, LIO-31321, LIO-79951) and the Janne Elgqvist Family Foundation. 


\section{REFERENCES}

Agüero-Torres, H., Thomas, V. S., Winblad, B., \& Fratiglioni, L. (2002). The impact of somatic and cognitive disorders on the functional status of the elderly. Journal of Clinical Epidemiology, 55, 1007-1012.

Allaire, J. C., Gamaldo, A., Ayotte, B. J., Sims, R., \& Whitfield, K. (2009). Mild Cognitive Impairment and objective instrumental everyday functioning: the Everyday Cognition Battery Memory Test. Journal of American Geriatrics Society, 57, 120-125.

Andersen, C.K., Wittrup-Jensen, K.U., Lolk, A., Andersen, K., \& Kragh-Sørensen, P. (2004). Ability to perform activities of daily living is the main factor affecting quality of life in patients with dementia. Health and Quality of Life Outcomes, 2, 52. doi:10.1186/1477$7525-2-52$

Andrén, E., Daving, Y., \& Grimby, G. (1997). Instrumental Activity Measure. Göteborgs University.

Aretouli, E., \& Brandt, J. (2009). Everyday functioning in mild cognitive impairment and its relationship with executive cognition. International Journal of Geriatric Psychiatry, 25, 224-233.

Bergdahl, E., Gustavsson, J. M. C., Kallin, K., von Heideken Wågert, P., Lundman, B., Bucht, G., \& et al. (2005). depression among the oldest old: the Umeå 85+ study. International Psychogeriatrics, 17 (4), 557-575.

Brooks, R. (1996). EuroQol: the current state of play. Health Policy, 37 (1), 53-72.

Burton, C. L., Strauss, E., Bunce, D., Hunter, M. A., \& Hultsch. D. F. (2009). Functional abilities in older adults with Mild Cognitive Impairment. Gerontology, 55, 570-581.

Cruz Moreira de Castro, K., \& Oliveira Guerra, R. (2008). Impact of cognitive performance on the functional capacity of an elderly population in Natal, Brazil. Arquivos de NeuroPsiquiatra, 66 (4), 809-813.

Daving, Y., Claesson, L., \& Sunnerhagen, K. S. (2009). Agreement in activities of daily living performance after stroke in a postal questionnaire and interview of communityliving persons. Acta Neurologica Scandinavica, 119 (6), 390-396.

Demura, S., Sato, S., Minami, M., \& Kasuga, K. (2003). Gender and age differences in basic ADL ability on the elderly: comparison between the independent and the dependent elderly. Journal of Oshysiologicalanthropology and Applied Human Science, 22 (1), 1927. 
Dodge, H. H., Kadowaki, T., Hayakawa, T., Yamakawa, M., Sekikawa, A., \& Ueshima, H. (2005). Cognitive impairment as a strong predictor of incident disability in specific ADLIADL tasks among community-dwelling elders: the Azuchi study. The Gerontologist, 45 (2), 222-230.

Dolan, P. (1997). Modeling valuations for EuroQol health states. Medical Care, 35, 10951108.

Dutton, D. B., \& Levine, S. (1989). Socioeconomic status and health: Overview, methodological critique, and reformulation. In: Bunker, J. P., Gomby, D. S., \& Kehrer, B. H., (Eds), Pathways to health: The role of social factors. 1st ed. Henry J Kaiser Family Foundation: Menlo Park, CA, p. 29-69.

EuroQol Group. (1990). EuroQol: A new facility for the measurement of health-related quality of life. Health Policy, 16, 199-208.

Folstein, M. F., Folstein, S. E., \& McHugh, P. R. (1975). Mini-Mental State: a practical method for grading the cognitive state of patients for the clinician. Journal of Psychiatric Research, 12 (3), 189-98.

Folstein, M. F., Folstein, S. E., McHugh, P. R., \& Fanjiang, G. (2001). Mini-Mental State Examination User's guide. Odessa, FL: Psychological Assessment Resources.

Gauthier, S., Reisberg, B., Zaudig, M., Petersen, R. C., Ritchie, K., Broich, K. et al. (2006). Mild cognitive impairment. Lancet, 367, 1262-1270.

Holland, R., Smith, R. D., Harvey, I., Swift, L., \& Lenaghan, E. (2004). Assessing quality of life in the elderly: a direct comparison of the EQ-5D and AQoL. Health Economics, 13, 793-805.

Johnson J. A., \& Pickard, A. S. (2000). Comparison of the EQ-5D and SF-12 health surveys in a general population survey in Alberta, Canada. Medical Care, 38 (1), 115-121.

Kurz, X., Scuvee-Moreau, J., Vernooij-Dassen, M., Dresse, A., \& the NADES Group. (2003). cognitive impairment, dementia and quality of life in patients and caregivers, Acta Neurologica Belgica, 103, 24-34.

König, H-H., Heider, D., Lehnert, T., Riedel-Heller, S. G., Angermeyer, M. C., Matschinger H., et al. (2010). Health status of the advanced elderly in six European countries: results from a representative survey using EQ-5D and SF-12. Health and Quality of Life Outcomes, 8, 143. doi:10.1186/1477-7525-8-143

Larsson, K., \& Thorslund, M. (2006). Chapter 8: Old people's health. Scandinavian Journal of Public Health, 34 (Suppl. 67), 185-198. 
Nygård, L. (2003). Instrumental activities of daily living: a stepping-stone towards Alzheimer's disease diagnosis in subjects with mild cognitive impairment? Acta Neurologica Scandinavica, 107 (Suppl. 179), 42-46.

Nygård, L., Amberla, K., Bernspång, B., Almkvist, O., \& Winblad, B. (1998). The relationship between cognition and daily activities in cases of mild Alzheimer's disease. Scandinavian Journal of Occupational Therapy, 5, 160-166.

Palmer, K., Fratiglioni, L., \& Winblad, B. (2003). What is mild cognitive impairment? Variations in definitions and evolution of non-demented persons with cognitive impairment. Acta Neurologica Scandinavica, 107 (Suppl. 179), 14-20.

Perneczky, R., Pohl, C., Sorg, C., Hartmann, J., Tosic, N., Grimmer, T. et al. (2006a). Impairment of activities of daily living requiring memory or complex reasoning as part of the MCI syndrome. International Journal of Geriatric Psychiatry, 21, 158-162.

Perneczky, R., Pohl, C., Sorg, C., Hartmann, J., Komossa, K., Alexopoulus, P. et al. (2006b). Complex activities of daily living in mild cognitive impairment; conceptual and diagnostic issues. Age and Ageing, 35, 240-245.

Polidoro, A., Dornbusch, T., Vestri, A., Di Bona, S., \& Alessandri, C. (2011). Frailty and disability in the elderly: A diagnostic dilemma. Archives of Gerontology and Geriatrics, 52, e75-e78.

Rabin, R., \& de Charro, F. (2001). EQ-5D: a measure of health status from the EuroQol Group. Annals of Medicine, 33, 337-343.

Thorvaldsson, V., MacDonald, S. W. S., Fratiglioni, L., Winblad, B., Kivipelto, M., Jonsson Laukka, E., \& et al. (2011). Onset and rate of cognitive change before dementia diagnosis: findings from two Swedish population-based longitudinal studies. Journal of the International Neuropsychological Society, 17, 1554-162.

Waldorff, F.B., \& Rishøj, S. (2008). I you don’t ask (about memory), they probably won’t tell. Journal of Family Practice, 57 (1), 41-44.

Wolfs, C.A.G., Dirksen, C.D., Kessels, A., Willems, D.C.M., Verhey, F.R.J., \& Severens, J.L. (2007). Performance of the EQ-5D and the EQ-5D+C in elderly patients with cognitive impairments. Health and Quality of Life Outcomes,5, 33. doi:10.1186/1477-7525-5-33 
Table 1. Characteristics of participants in relation to MMSE score, number (\%), and p-value

\begin{tabular}{|c|c|c|c|c|}
\hline & $\begin{array}{c}\text { MMSE 6-20 } \\
n=17\end{array}$ & $\begin{array}{c}\text { MMSE } 21-26 \\
n=91\end{array}$ & $\begin{array}{c}\text { MMSE 27-30 } \\
n=265\end{array}$ & $p$ \\
\hline Gender & & & & .775 \\
\hline Female & $11(65)$ & $51(56)$ & $156(59)$ & \\
\hline Male & $6(35)$ & $40(44)$ & $109(41)$ & \\
\hline Housing & & & & $<.001$ \\
\hline Ordinary housing & $9(53)$ & $79(87)$ & $254(96)$ & \\
\hline Community housing & $8(47)$ & $12(13)$ & $11(4)$ & \\
\hline Socioeconomic status (SES) & & & & $<.001$ \\
\hline Low & $15(88)$ & $58(65)$ & $117(45)$ & \\
\hline Intermediate & $1(6)$ & $23(26)$ & $122(47)$ & \\
\hline High & $1(6)$ & $8(9)$ & $22(8)$ & \\
\hline \multicolumn{5}{|l|}{ Assistance } \\
\hline Community assistance, daily & $6(35)$ & $16(18)$ & $17(6)$ & $<.001$ \\
\hline Transportation service & $11(65)$ & $41(46)$ & $81(31)$ & $<.001$ \\
\hline Personal alarm & $10(59)$ & $45(50)$ & $81(31)$ & $<.001$ \\
\hline \multicolumn{5}{|l|}{ Mobility assistive technology } \\
\hline Wheelchair & $3(18)$ & $8(9)$ & $9(3)$ & $.008 *$ \\
\hline Walker & $8(47)$ & $40(44)$ & $96(36)$ & .296 \\
\hline \multicolumn{5}{|l|}{$E Q-5 D$ items } \\
\hline Mobility problems & $10(59)$ & $56(62)$ & $109(41)$ & .002 \\
\hline Need help self-care & $8(47)$ & $17(19)$ & $18(7)$ & $<.001$ \\
\hline Need help usual activities & $11(65)$ & $36(40)$ & $40(15)$ & $<.001$ \\
\hline Pain/discomfort & $9(53)$ & $61(67)$ & $179(68)$ & .463 \\
\hline Anxiety/depression & $8(47)$ & $39(43)$ & $83(31)$ & .007 \\
\hline$E Q-5 D$ index value, mean (SD) & $0.58(0.36)$ & $0.68(0.25)$ & $0.75(0.22)$ & $.007 \dagger$ \\
\hline$E Q-5 D V A S$, mean $(\mathrm{SD})$ & $50.85(15.65)$ & $63.84(21.30)$ & $69.79(17.47)$ & $<.001 \dagger$ \\
\hline
\end{tabular}

Chi-squared test

* Fisher's exact test, comparison between two groups (impairment MMSE (6-20) vs. no impairment MMSE (27-30))

$\dagger$ Mann-Whitney U-test 
Table 2. Needing assistance in ADL in relation to MMSE score, number (\%), and p-value

\begin{tabular}{|c|c|c|c|c|}
\hline & $\begin{array}{l}\text { MMSE 6-20 } \\
\quad n=17\end{array}$ & $\begin{array}{c}\text { MMSE } 21-26 \\
n=91\end{array}$ & $\begin{array}{c}\text { MMSE 27-30 } \\
n=265\end{array}$ & $p$ \\
\hline \multicolumn{5}{|l|}{$P A D L$ items } \\
\hline Bath/take a shower & $12(71)$ & $19(21)$ & $23(9)$ & $<.001$ \\
\hline Dressing/undressing & $8(47)$ & $10(11)$ & $14(5)$ & $<.001$ \\
\hline Toileting & $4(24)$ & $3(3)$ & $3(1)$ & $.008 *$ \\
\hline Eating & $0(-)$ & $2(2)$ & $0(-)$ & NA \\
\hline \multicolumn{5}{|l|}{ IADL items } \\
\hline Locomotion outdoors & $9(53)$ & $49(54)$ & $109(41)$ & .086 \\
\hline Simple meal & $11(65)$ & $26(29)$ & $24(9)$ & $<.001$ \\
\hline Cooking & $11(65)$ & $44(48)$ & $51(19)$ & $<.001$ \\
\hline Public transportation & $12(71)$ & $50(55)$ & $83(31)$ & $<.001$ \\
\hline Small-scale shopping & $14(82)$ & $45(50)$ & $54(20)$ & $<.001$ \\
\hline Large-scale shopping & $15(88)$ & $55(60)$ & 87 (33) & $<.001$ \\
\hline Cleaning & $13(77)$ & $64(70)$ & $126(48)$ & $<.001$ \\
\hline Washing & $15(88)$ & $50(55)$ & $77(29)$ & $<.001$ \\
\hline
\end{tabular}

Chi-squared test

* Fisher's exact test, comparison between two groups (impairment MMSE (6-20) vs. no impairment MMSE (27-30))

$\mathrm{NA}=$ Not applicable 
Table 3. Correlations between MMSE score and IADL, r, level of significance

\begin{tabular}{ll}
\hline & MMSE \\
\hline Locomotion outdoors & $0.123 *$ \\
Simple meal & $0.321 * * *$ \\
Cooking & $0.331 * * *$ \\
Public transportation & $0.258 * * *$ \\
Small-scale shopping & $0.334 * * *$ \\
Large-scale shopping & $0.349 * * *$ \\
Cleaning & $0.269 * * *$ \\
Washing & $0.322 * * *$ \\
\hline
\end{tabular}

Spearman's rank correlation test

$* p<0.05$

$* * p<0.01$

$* * * \mathrm{p}<0.001$ 